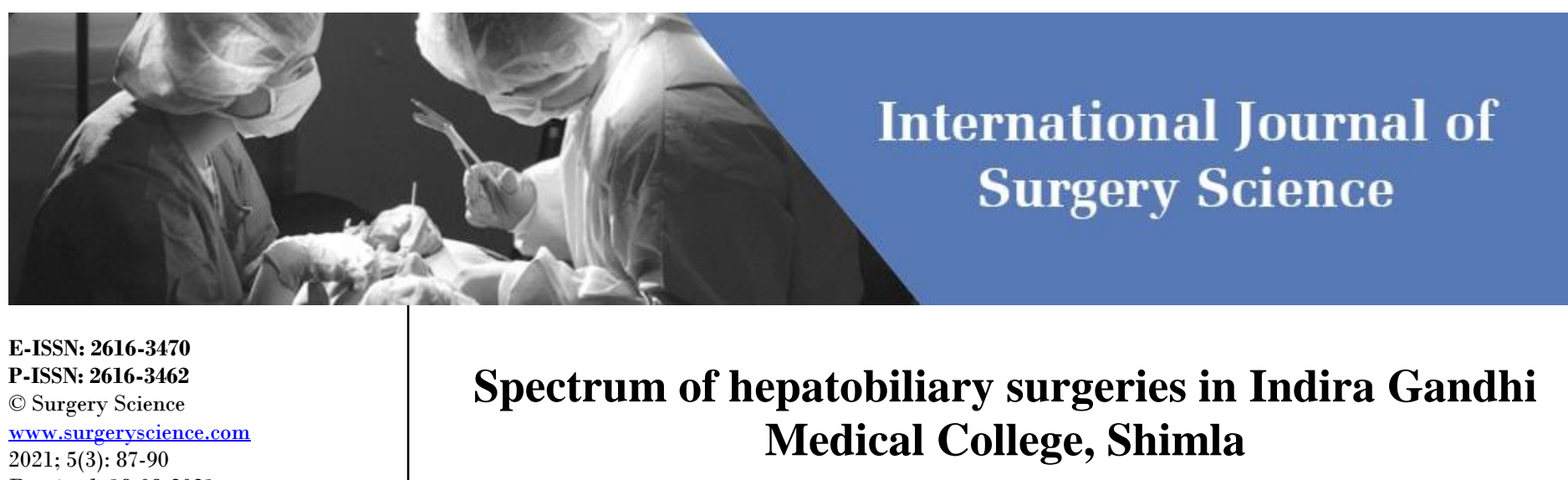

Received: 18-08-2021

Accepted: 23-09-2021

Dr. Rizul Prasher

Junior Resident Department of General Surgery IGMC, Shimla,

Himachal Pradesh, India

\section{Dr. DK Verma}

Professor and Head of

Department, Department of

General Surgery IGMC, Shimla,

Himachal Pradesh, India

\section{Dr. Gopal Singh}

Associate Professor, Department of General Surgery IGMC, Shimla,

Himachal Pradesh, India

\section{Dr. Arun Chauhan}

Associate Professor, Department of General Surgery IGMC, Shimla,

Himachal Pradesh, India

\section{Dr. Rashpal Singh}

Assistant Professor and Oncosurgeon Department of General Surgery IGMC, Shimla, Himachal Pradesh, India
Corresponding Author: Dr. Rizul Prasher Junior Resident Department of General Surgery IGMC, Shimla, Himachal Pradesh, India

\section{Dr. Rizul Prasher, Dr. DK Verma, Dr. Gopal Singh, Dr. Arun Chauhan and Dr. Rashpal Singh}

DOI: $\underline{\text { https://doi.org/10.33545/surgery.2021.v5.i4b.766 }}$

\section{Abstract}

Majority of the hepato-biliary surgeries are performed for benign conditions of all the common benign conditions, surgeries for gall stone disease makes the major share of surgeries. Malignant conditions comprise less than $5 \%$ of the hepato-biliary diseases. We intended to do a specific study in a tertiary care centre in IGMC, Shimla to ascertain the burden of diseases and its surgical management.

The procedures covered both benign and malignant conditions. Total number of elective surgeries performed during the study period were 7680 , out of which $5278(68.7 \%)$ belong to hepato-biliary surgeries. 5226 surgeries were performed for benign and 52 were performed for malignant diseases.

Laparoscopic cholecystectomy was the most commonly performed surgery. It is to be ascertained and requires further validation as to why such number of abnormally high cases of gall stone disease is prevalent in our institute and so is the incidence of gall bladder malignancy.

Keywords: Bladder malignancy, hepato-biliary, surgeries

\section{Introduction}

Hepato-biliary surgeries are designed to treat liver, bile duct and pancreatic pathologies which may be benign or malignant. These conditions are usually treated by hepato-biliary surgeons or are frequently managed by general surgeons. With better understanding of anatomy, the refinement in surgical techniques and reinforced by new imaging technologies, there has been a dramatic change in the morbidities and mortalities. Majority of the surgeries are performed for benign conditions which comprises of significant number of cases. Of all the common benign conditions, surgeries for gall stone disease makes the major share of surgeries, other less common conditions include surgeries for hydatid cyst of liver, common bile duct stones, choledochal cyst, pancreatic pseudocyst, benign neoplasm of pancreas and rarely surgery for acalculous cholecystitis and its complications and hepatic liver abscesses. Malignant conditions comprise less than $5 \%$ of the hepato-biliary diseases. We employed different surgical modalities as per the organ and its pathology involved. We intended to do a specific study in a tertiary care centre in IGMC, Shimla from June 2018 to July 2020 to ascertain the burden of diseases and its surgical management.

\section{Material and Methods}

A prospective observational study was conducted in Department of General Surgery, Indira Gandhi Medical College, and Shimla taking cases of hepatobiliary surgeries from 2018 to 2020. We performed different types of surgeries as per clinical diagnosis. Hepatobiliary surgeries included laparoscopic and open surgeries and documented all the procedures performed upon all the patients included in the study and recorded relevant points. Patients were thoroughly evaluated by taking a detailed history and performing detailed examination. All routine blood investigations and imaging were performed. Specific investigations in the form of CECT abdomen/MRI abdomen with MRCP were performed as per hepato-biliary protocol. All the patients undergoing major surgical resections pre-operatively were optimized and then subjected to different types of surgical procedures. Detailed surgical procedures and findings of each patient were documented and specimen labelled and sent for histopathological examination. Patients were followed up for any post-operative complications and recurrence. 


\section{Inclusion Criteria}

All new patients admitted in the department of General Surgery with hepatobiliary diseases posted for surgery.

\section{Exclusion Criteria}

1) Patients admitted with hepato-biliary diseases, not posted for surgery

2) Patients unfit for surgery

\section{Observations}

The study includes a total of 5278 cases/patients that were studied prospectively (June 2018 - July 2020). The following observations are made:

Gall bladder disease was the most common pathology operated upon accounting for 5095 number $(96.5 \%)$ of total cases followed by liver disorders with $99(1.9 \%)$, Biliary tract disorders with $75(1.4 \%)$ and pancreatic disorders with $9(0.2 \%)$ of the total cases/patients as shown in table1.

Table 1: Frequency of Hepato-biliary and pancreatic cases which were operated $(n=5278)$

\begin{tabular}{|c|c|c|}
\hline Hepato-biliary and pancreatic disorders & Number of cases & Percentage \\
\hline \multicolumn{3}{|l|}{ Gall bladder disease } \\
\hline - Symptomatic cholelithiasis & 4702 & 89 \\
\hline - Empyema gall bladder & 112 & 2.1 \\
\hline - Mucocele gall bladder & 252 & 4.8 \\
\hline - Carcinoma gall bladder & 29 & 0.5 \\
\hline Total & 5095 & 96.5 \\
\hline \multicolumn{3}{|l|}{ Liver disorders } \\
\hline - Hydatid cyst liver & 99 & 1.9 \\
\hline \multicolumn{3}{|l|}{ Biliary tract disorders } \\
\hline - Cholelithiasis with choledocholithiasis & 55 & 1 \\
\hline - Periampullary carcinoma & 17 & 0.3 \\
\hline - Bile duct strictures & 2 & 0.03 \\
\hline - Choledochal cyst & 1 & 0.01 \\
\hline Total & 75 & 1.4 \\
\hline \multicolumn{3}{|l|}{ Pancreatic disorders } \\
\hline - Pancreatic carcinoma & 6 & 0.1 \\
\hline - Pseudocyst of pancreas & 2 & 0.03 \\
\hline - Acute pancreatitis with necrosis & 1 & 0.01 \\
\hline Total & 9 & 0.2 \\
\hline Grand total & 5278 & 100 \\
\hline
\end{tabular}

In hepato-biliary and pancreatic disorders most common indication for surgery was symptomatic cholelithiasis and most commonly performed procedure was laparoscopic cholecystectomy accounting for $79 \%$ followed by open cholecystectomy accounting for $15.2 \%$ surgeries and single incision laparoscopic cholecystectomy accounting for $1.9 \%$ of surgeries. In patients with cholelithiasis and choledocholithiasis most commonly performed surgery was open cholecystectomy with choledochoduodenostomy accounting for $0.8 \%$ of surgeries followed by open cholecystectomy with T-tube drainage of CBD accounting for $0.15 \%$ of surgeries. $0.06 \%$ of laparoscopic CBD exploration were also done.

For all cases of bile duct strictures hepaticojejunostomy was performed accounting for $0.03 \%$ and for choledochal cyst, excision of cyst with hepaticojejunostomy was performed accounting for $0.01 \%$ of cases.

For hydatid cyst of liver most commonly performed procedure was enucleation with capitonnage of cyst cavity accounting for $1.0 \%$ of surgeries followed by enucleation with marsupialization of cyst and external drainage accounting for $0.6 \%$ and in cases of $\mathrm{CBC}$, enucleation with primary repair of $\mathrm{CBC}$ with external drainage was performed accounting for $0.2 \%$ of surgeries.

For pseudocyst pancreas, cystogastrostomy was performed accounting for $0.03 \%$ cases and necrosectomy was performed for acute necrotizing pancreatitis accounting for $0.01 \%$ cases.

In cases of periampullary carcinoma most commonly performed surgery was Whipple's procedure accounting for $0.4 \%$ of total hepato-biliary surgeries and for carcinoma pancreas involving distal part, distal pancreatectomy with splenectomy was performed accounting for $0.03 \%$ of cases. Triple bypass was performed in $0.03 \%$ cases of carcinoma head of pancreas as a palliative procedure.

For carcinoma gall bladder, extended cholecystectomy was performed accounting for $0.5 \%$ of surgeries with completion extended cholecystectomy for patients who had undergone cholecystectomy previously and gall bladder turned out to be carcinoma gall bladder on HPE, accounting for $0.03 \%$ of surgeries (Table 2).

Table 2: Accounting for $0.03 \%$ of surgeries

\begin{tabular}{|c|c|c|c|c|}
\hline Procedure & Disease & Modalities of Treatment Performed & Number of cases & Percentage \\
\hline \multirow{4}{*}{ Cholecystectomy } & \multirow{4}{*}{$\begin{array}{l}\text { Symptomatic } \\
\text { cholelithiasis }\end{array}$} & $\mathrm{L} / \mathrm{C}$ & 4160 & 79.0 \\
\hline & & 2. $\mathrm{O} / \mathrm{C}$ & 807 & 15.2 \\
\hline & & 3. $\quad$ SILC & 99 & 1.9 \\
\hline & & Total & 5066 & 96.0 \\
\hline \multirow{4}{*}{$\begin{array}{l}\text { Open cholecystectomy with } \\
\text { common bile duct } \\
\text { exploration }\end{array}$} & \multirow{4}{*}{$\begin{array}{l}\text { Cholelithiasis with } \\
\text { choledocholithiasis }\end{array}$} & 1. Laparoscopic CDL with primary repair & 3 & 0.06 \\
\hline & & 2. O/C with CDL with CDD & 44 & 0.8 \\
\hline & & 3. $\mathrm{O} / \mathrm{C}$ with $\mathrm{CDL}$ with $\mathrm{T}$-tube drainage & 8 & 0.15 \\
\hline & & Total & 55 & 1.0 \\
\hline Hepaticojejunostomy & Bile duct strictures & Hepaticojejunostomy & 2 & 0.03 \\
\hline Excision of choledochal cyst & Choledochal cyst & Excision of choledochal cyst with hepaticojejunostomy & 1 & 0.01 \\
\hline
\end{tabular}




\begin{tabular}{|c|c|c|c|c|}
\hline \multicolumn{5}{|l|}{ with hepaticojejunostomy } \\
\hline \multirow{4}{*}{$\begin{array}{c}\text { Enucleation with } \\
\text { management of residual } \\
\text { cavity }\end{array}$} & \multirow{4}{*}{ Hydatid cyst liver } & 1. Enucleation with capitonnage of cyst cavity & 55 & 1.0 \\
\hline & & 2. $\quad$ Enucleation with marsupialization of cyst \& external drainage & 32 & 0.6 \\
\hline & & 3. $\quad$ Enucleation with repair of CBC and external drainage & 12 & 0.2 \\
\hline & & Total & 99 & 1.9 \\
\hline Cystogastrostomy & $\begin{array}{l}\text { Pseudocyst } \\
\text { pancreas }\end{array}$ & Cystogastrostomy & 2 & 0.03 \\
\hline Necrosectomy & $\begin{array}{c}\text { Acute necrotizing } \\
\text { pancreatitis }\end{array}$ & Necrosectomy & 1 & 0.01 \\
\hline \multirow{4}{*}{ Pancreatic surgeries } & \multirow{4}{*}{$\begin{array}{c}\text { Periampullary and } \\
\text { pancreatic } \\
\text { carcinoma }\end{array}$} & Whipple's procedure & 19 & 0.4 \\
\hline & & Distal pancreatectomy with splenectomy & 2 & 0.03 \\
\hline & & Triple bypass & 2 & 0.03 \\
\hline & & Total & 23 & 0.4 \\
\hline \multirow{4}{*}{$\begin{array}{l}\text { Extended and completion } \\
\text { cholecystectomy }\end{array}$} & \multirow{3}{*}{$\begin{array}{l}\text { Carcinoma gall } \\
\text { bladder }\end{array}$} & 1. Extended cholecystectomy & 27 & 0.5 \\
\hline & & 2. Completion cholecystectomy & 2 & 0.03 \\
\hline & & Total & 29 & 0.5 \\
\hline & & Grand total & 5278 & 100.0 \\
\hline
\end{tabular}

\section{Discussion}

In our institution total number of elective surgeries performed during the study period were 7680 , out of which $5278(68.7 \%)$ belong to hepato-biliary surgeries. On reviewing the literature, our study number for hepato-biliary surgeries was in contradiction to the hepato-biliary surgeries performed by various specialized centres. Our being a non-specialized centre, accommodates all the types of surgeries. The number of surgeries being performed for benign diseases like gall stone disease was significantly high whereas specialized centres entertain the cases of complex hepato-biliary surgeries only.

We performed all types of hepato-biliary surgeries except nonoperable or unfit patients. These procedures covered both benign and malignant conditions. Total of 5226 surgeries were performed for benign diseases and 52 were performed for malignant diseases.

The most common disorder was symptomatic cholelithiasis accounting for $89 \%$ of all hepato-biliary disorders operated, followed by empyema gall bladder and mucocele gall bladder accounting for $2.1 \%$ and $4.8 \%$ cases respectively. The most common surgery performed for symptomatic cholelithiasis was laparoscopic cholecystectomy accounting for $79 \%$ of hepatobiliary surgeries, followed by open cholecystectomy performed in $15.2 \%$ cases and single incision laparoscopic cholecystectomy (SILC) performed in $1.9 \%$ of cases. Similar results were shown by Swaraj Sambit et al. ${ }^{[11]}$. They performed 67\% laparoscopic cholecystectomies and 30\% open cholecystectomies. Robert E. Glasgow et al. in their study showed that ratio of $\mathrm{L} / \mathrm{C}$ to $\mathrm{O} / \mathrm{C}$ was 10.2:1 in uncomplicated gall stone disease and 2.3:1 in complicated gall stone disease ${ }^{1}$. Robert E. Glasgow et al. and Arpit Bansal et al. concluded in their studies that for gall stone disease laparoscopic cholecystectomy should be preferred and is the procedure of choice ${ }^{[1,2]}$. As more and more surgeons are now familiar with laparoscopic techniques, majority of gall stone diseases are being managed by laparoscopic techniques and conversion rate has also gone down. There is high rate of laparoscopic cholecystectomy in our institute as probably there is high incidence of gall stone disease in Himachal Pradesh. We need to look for possible causes for high burden of gall stone disease in our area.

In present study for carcinoma gall bladder, extended cholecystectomy was performed in all operable cases. It is the most common procedure performed for malignant diseases of hepato-biliary system. Abdul Kaiyum Khan et al. and Vikas Ostwal et al. showed similar results in their studies. They concluded that good quality optimal surgery, avoidance of unnecessarily morbid surgeries, the use of radical/extended cholecystectomy in patients with disease beyond T1a stage, is the surgical management of choice in resectable cases of carcinoma gall bladder ${ }^{[3,4]}$. Due to high incidence of gall stone disease, there is increased predisposition to carcinoma gall bladder. This requires further studies for validation.

Cholelithiasis with choledocholithiasis constituted $1 \%$ of total operated patients. Depending upon the number, size of CBD stones and diameter of $\mathrm{CBD}$, various surgical procedures were performed. The most common surgery performed was choledocholithotomy with choledochoduodenostomy comprising of $0.8 \%$ of total surgeries performed on hepato-biliary system, $0.15 \%$ open cholecystectomy with choledocholithotomy with $\mathrm{T}$ tube drainage of common bile duct and $0.06 \%$ laparoscopic CBD exploration was done. Purujit Choudhury et al. conducted a study regarding the surgical management of CBD stones, open choledochotomy with T-tube drainage was done in majority of patients ${ }^{5}$. Another study conducted by Abolfazl Shojaiefard on various techniques for management of $\mathrm{CBD}$ stones reported that laparoscopic CBD exploration (LCBDE) was the most common surgery performed in their institution and concluded that LCBDE (trans-cystic or trans-ductal) is a standard method with a high efficacy and low morbidity and mortality for the treatment of CBD stones in most centres but open approach always remains as the final option when other modalities have failed ${ }^{6}$. Patients referred to our department generally present with multiple CBD stones in our setting, so most commonly choledochoduodenostomy is performed. Laparoscopic CBD exploration is not commonly/routinely done in our institute due to lack of logistic support.

For all cases of bile duct strictures hepaticojejunostomy was performed accounting for $0.03 \%$ and for choledochal cyst, excision of cyst with hepaticojejunostomy was performed accounting for $0.01 \%$ of cases.

In the present study hydatid cyst liver accounted for $1.9 \%$ of cases operated for hepato-biliary surgeries. The most common surgery performed was enucleation with capitonnage of cyst cavity accounting for $1 \%$ of cases, followed by enucleation with marsupialisation with external drainage of cyst cavity accounting for $0.6 \%$ of cases. Patients who had CBC were operated with enucleation with repair of $\mathrm{CBC}$ with external drainage of hydatid cyst cavity accounting for $0.2 \%$ of cases. The choice of surgical technique and the radical procedure involved is influenced by size, site, type of the cyst, complications, relation to bile ducts and blood vessels, clinical presentation and surgical expertise. In general, surgical excision was the preferred treatment option. Sanjay Goja et al., in their study on hydatid cyst treatment reported that 5 patients 
underwent partial cystectomy (2 laparoscopic and 3 open), 9 cysto-pericystectomy (7 open and 2 robotic) and 7 hepatectomies (1 central, 4 right, 1 left and 1 right trisectionectomy). Living donor liver transplantation was performed in 3 patients with alveolar echinococcus disease and the fourth patient underwent right trisectionectomy with en-bloc resection of hepatic flexure and right diaphragm ${ }^{[8]}$. Guillaume Martel et al. conducted a study on surgical management of symptomatic hydatid liver disease and reported that most patients in their centre $(83 \%)$ underwent liver resection with or without drainage/marsupialization. Radical liver resection was carried out in 60\% (19 major, 5 minor) ${ }^{[7]}$. Laparoscopic hydatid cyst surgeries are not performed in our institute. Hepatectomy and radical liver resections are not performed in our institute due to lack of facilities and trained surgeons for complex vascular reconstruction and post-operative dedicated ICU.

Pseudocyst pancreas accounted for $0.03 \%$ cases and surgery performed was cystogastrostomy in all cases. Acute necrotizing pancreatitis was diagnosed in $0.01 \%$ cases and necrosectomy was done in that case.

In the present study, periampullary carcinoma accounted for $0.3 \%$ of hepato-biliary cases. In the present study, for resectable tumours, all patients underwent Whipple's procedure which accounted for $0.4 \%$ of cases for resectable tumours. Similar results were reported by Chaobin $\mathrm{He}$ et al., who showed that resection was indicated in operable and resectable cases, classical Whipple's operation was the standard resection performed [9]. Francesca Panzeri et al. also reported that in ampullary carcinoma, pancreaticoduodenectomy (PD), either with conventional or pylorus-preserving approach (PPPD), is considered the standard of care and was performed in all patients who were resectable and operable ${ }^{[10]}$.

In the present study carcinoma pancreas accounted for $0.1 \%$ of cases and surgery performed for growth in head and body region was Whipple's procedure in resectable cases. For growth in distal part of pancreas, distal pancreatectomy with splenectomy was performed in all resectable cases and it accounted for $0.03 \%$ of all hepato-biliary surgeries. Triple bypass was performed as a palliative procedure in $0.03 \%$ of cases.

\section{Conclusion}

It is concluded that laparoscopic cholecystectomy was the most commonly performed surgery for hepatobiliary diseases in our centre. It is to be ascertained and requires further validation as to why such number of abnormally high cases of gall stone disease is prevalent in our institute and so is the incidence of gall bladder malignancy. Our centre is responsible for all types of surgical ailments and hepato-biliary diseases takes the lion's share. A host of hepato-biliary pathologies ranging from benign such as cholelithiasis to malignant pathologies such as cancers of liver, pancreas and biliary tract affect people of all ages. A significant load of such patients is present in our institute as it is a tertiary level care center of the state. It is always a great challenge to provide needy patients with the best possible surgical care in a resource limited healthcare facility. With dedicated surgeons, staff, operation theatres, wards and ICU more complex hepatobiliary surgeries like hepatic resections, liver transplant, Whipple's procedure with vascular reconstruction can be performed after the development of required infrastructure and training for the surgeons and supporting staff. Thus, the need of the hour is the establishment of a separate hepatobiliary unit at our institute to handle the load of these surgeries and to provide optimum care.

\section{References}

1. Robert Glasgow E, Michael Chow, Matthew Hutter M et al. The spectrum and cost of complicated gall stone disease in California. Arch Surg 2000;135:1021-1027.

2. Arpit Bansal, Murtaza Akhtar, Ashwani Kumar Bansal. A clinical study: prevalence and management of cholelithiasis. Int Surg J 2014;1(3):134-139.

3. Abdul Kaiyum Khan, Rishin Dutta, Diptendra Kumar Sarkar et al. Spectrum and management of gall bladder carcinoma. International journal of scientific research 2020;9(12):8179-8183.

4. Vikas Ostwal, Sanyo Dsouza, Shraddha Patkar et al. Current management strategies in gallbladder cancers. Cancer Res Stat Treat 2018;1:2-9.

5. Dr Purujit Choudhury, Dr Aadish Goyal. The Clinical Spectrum of Choledocholithiasis with Special Reference to Different Surgical Management. Int J Health Sci Res 2019;9(1):152-157.

6. Abolfazl Shojaiefard, Majid Esmaeilzadeh, Ali Ghafouri et al. Various Techniques for the Surgical Treatment of Common Bile Duct Stones: A Meta Review. Gastroenterology Research and Practice 2009.

7. Guillaume Martel, Salima Ismail, André Bégin et al. Surgical management of symptomatic hydatid liver disease: experience from a Western centre. Can J Surg 2014;57(5):320-6.

8. Sanjay Goja, Sujeet Kumar Saha, Sanjay Kumar Yadav. Surgical approaches to hepatic hydatidosis ranging from partial cystectomy to liver transplantation. Ann Hepatobiliary Pancreat Surg 2018;22(3):208-215.

9. Chaobin He, Yize Mao, Jun Wang et al. Surgical management of periampullary adenocarcinoma: defining an optimal prognostic lymph node stratification schema. J Cancer 2018;9(9):1667-1679.

10. Francesca Panzeri, Stefano Crippa, Paola Castelli. Management of ampullary neoplasms: A tailored approach between endoscopy and surgery. World J Gastroenterol 2015;21(26):7970-7987.

11. Swaraj Sambit Samal, Manas Ranjan Behera, Subhabrata Das et al. A Study on Prevalence, Clinical Presentation, and Management of Gall Stone Diseases in Southern Odisha. J Evid Based Med Health 2020;7(32):1628-1632. 